Research Article

\title{
Comparative Study of Coronary Artery Proximal to Myocardial Bridge Segment
}

\author{
Monika Srivastava', Asha Dixit ${ }^{2}$, Anuj]ain ${ }^{3}, \underline{\text { Vandana Sharma }}{ }^{4}$, Nisha Yadav $^{5}$, Manisha Pandey $^{6}$ \\ 1,3,5,6 Department of Anatomy, University of Medical Sciences, Saifai, Etawah, Uttar Pradesh, India. \\ ${ }^{2,4}$ Department of Anatomy, Gandhi Medical College, Bhopal, Madhya Pradesh, India.
}

DOI: https://doi.org/10.24321/2394.6539.202112

\section{I $\quad \mathbf{N} \quad \mathbf{F} \quad \mathbf{O}$}

\author{
Corresponding Author: \\ Monika Srivastava, University of Medical \\ Sciences, Saifai, Etawah, Uttar Pradesg, India. \\ E-mail Id: \\ monikasrivastava1981@gmail.com \\ Orcid Id: \\ https://orcid.org/0000-0003-2961-0980 \\ How to cite this article: \\ Srivastava M, Dixit A, Jain A, Sharma V, Yadav N, \\ Pandey M. Comparative study of Coronary Artery \\ Proximal to Myocardial Bridge Segment. J Adv \\ Res Med Sci Tech. 2021;8(3):5-9. \\ Date of Submission: 2021-07-20 \\ Date of Acceptance: 2021-09-02
}

\section{$\begin{array}{llllllll}\mathbf{A} & \mathbf{B} & \mathbf{S} & \mathbf{T} & \mathbf{R} & \mathbf{A} & \mathbf{C} & \mathbf{T}\end{array}$}

Introduction: The muscular segments which overlie the epicardial arteries are termed as myocardial bridges and the artery which travels through them are termed as tunnel arteries. These tunnel arteries get compressed during the systolic compression of the heart, thus partially or completely blocking the blood supply to the corresponding areas.

Aim \& Objectives: To assess the impact of these myocardial bridges on the proximal segment of the myocardial arteries.

Methodology: The present study was cadaveric-based cross-sectional study. A total of 22 hearts which showed the presence of myocardial bridges were collected from two sources namely: cadaver dissections, autopsy. The hearts were clean and numbered. This was followed by fixation, dehydration, clearing, embedding, block formation, section cutting and staining.

Result: The present study showed that there is a significant thickening in the tunica intima of the proximal to bridge segment of the coronary artery. The present study also noted that there is a marked thinning of the tunica media of the same segment.

Conclusion: The present study concludes that there is a marked hyperplasia in the proximal segment of the myocardial bridges under tunica intima.

Keywords: Myocardial Bridge, Proximal Segment of Myocardial bridge, Tunica Intima

\section{Introduction}

The muscular segment which overlies the epicardial arteries are termed as myocardial bridges and the artery which travels through them are termed as tunnel artery. (1) Epicardial arteries play an important role in providing oxygenation and blood supply to the muscles of heart. However, these tunnel arteries get compressed during the systolic compression of the heart, thus partially or completely blocking the blood supply to the corresponding areas.

Majority of the times, these are asymptomatic and remain silent. However, some patients may develop symptoms pertaining to chest pain or chest heaviness. The most common site of these myocardial bridges is left anterior descending and majority of times these are usually single but multiple bridge are also not uncommon. 


\section{Clinical Implications}

The incidence of myocardial bridges are reported to be in between $1.5 \%$ to $16 \% .{ }^{2}$ Majority of the times these are the congenital anomalies and are considered as benign in nature. However, recent studies have shown that they have some major clinical implications. Some of these implication are ischemia and acute coronary syndromes, ${ }^{3-8}$ coronary spasm, ${ }^{9}$ ventricular septal rupture, ${ }^{10}$ arrhythmias (including supraventricular tachycardia and ventricular tachycardia), ${ }^{11}$ exercise-induced atrioventricular conduction blocks, ${ }^{12}$ transient ventricular dysfunction, ${ }^{13}$ and sudden death. ${ }^{14}$

\section{Histological Changes}

Theoretically speaking, myocardial bridges fall into two major segments namely: the superficial segment which account for nearly $75 \%$ of all the cases which mostly lies in the intraventricular groove and a deep segment right ventricle and dives into the intraventricular septum. It is proposed that systolic compression of the superficial subtype happens only infrequently; however, the deep variant results in twisting of the tunnelled segment over the course of the cardiac cycle, resulting in hemodynamic compromise during coronary flow. ${ }^{15}$

There is a limited research which suggests the histological changes in the proximal segment of the same coronary arteries due to the impact of these myocardial bridges. So, the present study is designed to assess the impact of these myocardial bridges on the proximal segment of the myocardial arteries.

\section{Material and Method}

The present study was cadaveric-based cross-sectional study carried in the Department of Anatomy, Gandhi Medical College, Bhopal, MP, India. A total of 22 hearts which showed the presences of myocardial bridges were collected from two sources, namely, cadaver dissections, autopsy. These hearts were cleaned and numbered before keeping separately with all the aseptic precautions in $10 \%$ formalin.

The inclusion criteria were:

- Only adult hearts were used

- Bridges present only either of left or right coronary artery were included

\section{Methodology}

Once a myocardial bridge had been identified, it was cleaned properly and numbered. The exact locations from the beginning till the end of myocardial bridges were defined.

\section{Histological Technique}

Following histological technique was used in the processing of the tissue:

\section{Fixation and Dehydration}

Tissues were fixed in $10 \%$ formalin. Duration of fixation ranged from 24 to $48 \mathrm{hrs}$. This was followed by process of dehydration using ascending series of alcohol.

\section{Clearing/ Dealcoholisation}

Tissues were transferred into cedar wood oil till they become transparent. Time required for clearing was $2 \mathrm{hrs}$ to 10 days.

\section{Wax Impregnations}

After cleaning, tissues were transferred into fresh mottled wax in an open jar. Three changes of paraffin of 30-30 minutes in vacuum oven were required. Wax with a melting point of $56^{\circ} \mathrm{C}$ to $58^{\circ} \mathrm{C}$ was used.

\section{Embedding}

Suitable molten paraffin wax used. The specimens were molten in the mould such that cutting can be done at the right angle.

\section{Section Cutting}

With a sharp knife, correctly adjusted microtome, the blocks were sectioned. The thickness of sections taken varied from 8 to 10 microns. With the help of needle, sections were transferred to warm water. The cut sections were then mounted on a glass slide smeared with adhesive. The slides were thoroughly dried on the hot plate and stored in specially designed harvesting cabinet.

\section{Staining}

Routine staining of tissue sections were done with haematoxylin and eosin (H\&E).

Solution required were:

- Ripened Harris' haematoxylin solution

- $1 \%$ aqueous solution of eosin $Y$

- $70 \%$ alcohol

- $90 \%$ alcohol

- $95 \%$ alcohol

- Absolute alcohol

- Xylene

- Hydrocloric acid

- D.P.X (A synthetic resin medium)

- $\mathrm{DPX}=$ mixture of distrene ( a polystyrene), a plasticizer (tri-cresyl phosphate) and xylene

\section{Staining Techniques}

The following staining method was used to stain tissue section for light microscopic examination:

The slides were gently warmed (with paraffin sections) over hot plate and placed quickly in Xylene 1 for $2 \mathrm{~min}$, then xylene II for $2 \mathrm{~min}$. This caused removal of paraffin wax from sections.

Sections were placed in absolute alcohol I for $1 \mathrm{~min}$, then absolute II for $1 \mathrm{~min}$. Xylene was removed from section. 
Slides were treated with descending grade of alcohol as follows:

90\% alcohol - $2 \mathrm{~min}$

$70 \%$ alcohol - 2 min

- $\quad$ Rinsed briefly with running tap water

- Stained in Haematoxylin for 2-5 min. The sections took a blue color

- Washed well in running tap water for 2 to $3 \mathrm{~min}$; the sections were examined under microscope at this stage to confirm a sufficient degree of staining. If insufficient, sections were returned to the re-stain

- $\quad$ Excess stains were removed by differentiating in 0.5-1 $\%$ hydrochloric acid in $70 \%$ alcohol for a few seconds. The blue staining of Haematoxylin was changed to red by action of the acid

- To regain the blue color and stop decolorization, slides were washed in alkaline, running tap water for at least 5 min and examined under microscope to check staining

- $\quad$ Stained in $1 \%$ eosin for 1 to 3 min followed by rinsing in tap water to wash off surplus stain

- Sections were dehydrated in alcohol as follows:

95\% Alcohol - $2 \mathrm{~min}$

Absolute alcohol III - $2 \mathrm{~min}$

Absolute alcohol IV - 2 min

- Sections were cleared in xylene III for $2 \mathrm{~min}$

- Transferred the section to a final bath of xylene IV

- Mounted in DPX

\section{Staining Results}

Nuclei - blue to black colour.

Cytoplasm and muscle fibres - pink.

Table I.Showing the Comparison of Tunica Intima and Tunica Media in the Proximal to Bridge Segment of Coronary Artery with Normal Coronary Artery

\begin{tabular}{|c|c|c|c|c|}
\hline S. No. & Artery & Study & Control & P value \\
\hline 1. & $\begin{array}{c}\text { Tunica } \\
\text { intima }\end{array}$ & $\begin{array}{c}3.431 \pm \\
0.59\end{array}$ & $\begin{array}{c}1.04 \pm \\
0.31\end{array}$ & $\begin{array}{c}\mathrm{T}=16.87 \mathrm{df} 42 \\
\mathrm{p}=<0 . .1\end{array}$ \\
\hline 2. & Tunica & $\begin{array}{c}1.765 \pm \\
0.09\end{array}$ & $\begin{array}{c}2.27 \pm \\
1.03\end{array}$ & $\begin{array}{c}\mathrm{T}=2.291 \\
\mathrm{Df}-21.3 \\
\mathrm{P} \text { value }=0.032\end{array}$ \\
\hline
\end{tabular}

\section{Result}

The 22 hearts, obtained from 22 cadavers, showed the presence of myocardial bridges in single or both coronary arteries. All the 22 cadaveric hearts had myocardial bridge in Left Coronary Artery (LCA) in anterior intervertebral artery while only three cadaveric hearts showed the presence of myocardial bridge in the posterior intervertebral branch of Right Coronary Artery (RCA). In the left coronary artery
(LCA), five hearts showed two bridges while in right coronary artery showed single bridge.

On doing the histological evaluation of the coronary arteries, it was noted that there is a marked hyperplasia in both the tunica intima of the proximal to bridge segments of these arteries. Whereas there is a thinning in the tunica media of the same segment (Table 1) (Figures $1 \& 2$ ).

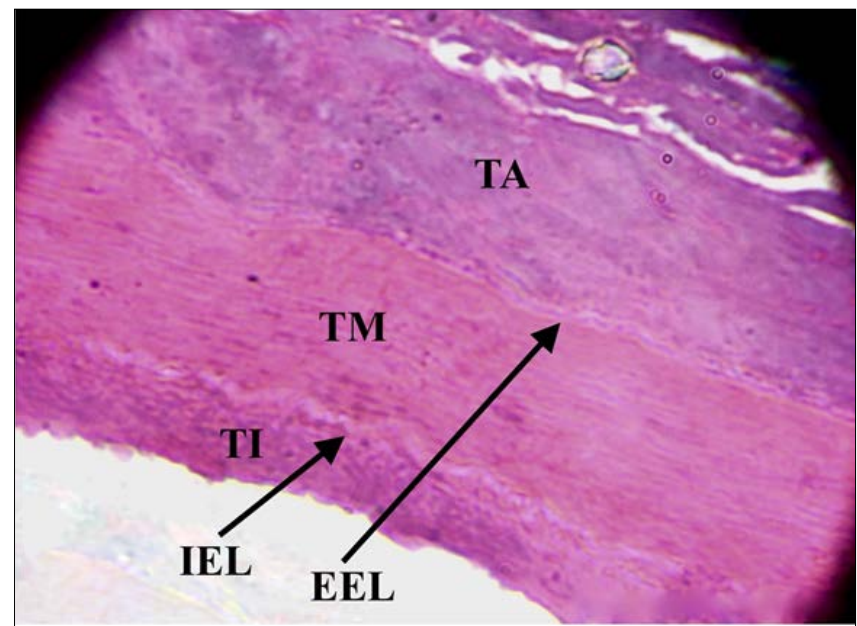

TA: Tunica Adventitia TM: Tunica Media TI: Tunica Intima EEL: External Elastic Lamina IEL: Internal Elastic Lamina

Figure I.Showing the Control Coronary Artery

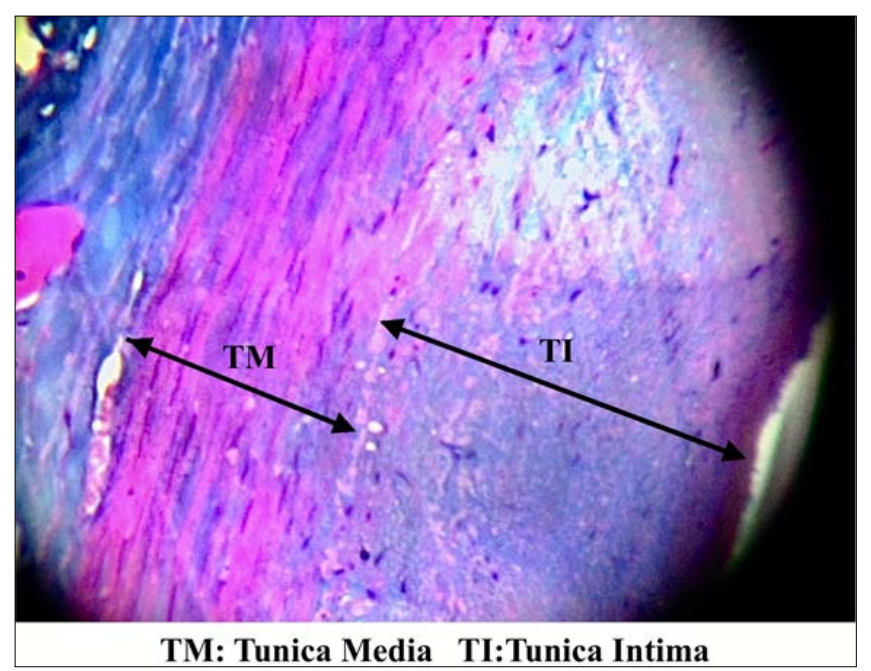

Figure 2.Showing the Proximal Segment of Bridge Coronary Artery

\section{Discussion}

Myocardial bridges are, the majority of time, accidental finding. ${ }^{16}$ However, they may present with a wide variety of symptoms. There are marked histological changes that take place during the course of the artery as it passes through the segment of the myocardial bridge. The present study highlighted the changes that take place in the proximal segment of the myocardial artery as it passes through the 
myocardial bridge. There is a marked hyperplasia in the tunica intima in the proximal to bridge segment of the coronary artery as compared to the control artery. This difference is statistically significant. Studies conducted by other researcher like Shinjo et al. ${ }^{17}$ and Saidi et al. ${ }^{18}$ have also noted similar intimal hyperplasia in their studies.

This intimal hyperplasia in the proximal segment accelerate the formation of atherosclerosis plaque. The mechanism of ischemia does not appear to be entirely related to this fixed obstruction. A portion of the effect relates to tachycardic states in which diastolic filling shortens and lessens in importance and disturbances in systolic filling have a greater effect. ${ }^{19}$ Studies based on angiography have also shown that vessel compression of bridged arteries extended into diastole and thus did affect the predominant phase of coronary perfusion. ${ }^{20}$

Studies conducted by Klue ${ }^{21}$ using Doppler flow and pressure measurement in patients with symptomatic bridges noted that there are hemodynamic abnormalities characterized by a persistent decrease in diastolic vessel diameter, increase in blood flow velocities and retrograde flow, and a reduced flow reserve.

It was also noted in the present study there is also a statistically significant thinning in the tunica media segment of the same proximal coronary artery as compared to the control artery. However, the clinical relevance of this has also to be studied.

It was noted that earlier researcher like Ge et $a^{22}$ the projective role of myocardial bridge in the underbridge segment from atherosclerotic plague formation. The intima of the tunnelled segment is significantly thinner than the proximal segment, and contains a predominance of the "contractile" subtype of smooth muscle cells thought to be negatively associated with the development of atherosclerotic lesions. Conversely, the vessel segment proximal to the bridge appears to develop atherosclerosis at increased rates, approaching $90 \% .^{14}$

\section{Conclusion}

The present study herby concludes that there is a marked hyperplasia in tunica intima proximal to bridge segment. However, there is also marked thinning of the tunica media of the same segment. The hyperplastic tunica intima facilitates the development of atherosclerotic plaque.

Patients present with unexplained chest pain should be investigated for the presence of myocardial bridges in them.

\section{Conflict of Interest: None \\ References}

1. Konen E, Goitein O, Di Segni E. Myocardial bridging, a common anatomical variant rather than a congenital anamaly. Semin Ultrasound CT MR. 2008;29:195-203. [Pubmed] [Google Scholar]

2. Rossi L, Dander B, Nidasio GP, Arbustini E, Paris B, Vassanelli C, Buonanno C, Poppi A. Myocardial bridges and ischemic heart disease. Eur Heart J. 1980;1:239-45. [PubMed] [Google Scholar]

3. Kneale BJ, Stewart AJ, Coltart DJ. A case of myocardial bridging: Evaluation using intracoronary ultrasound, Doppler flow measurement, and quantitative coronary angiography. Heart 1996;76:374-6. [PubMed] [Google Scholar]

4. Tauth J, Sullebarger T. Myocardial infarction associated with myocardial bridging: Case history and review of the literature. Cathet Cardiovasc Diagn. 1997;40:364-7. [PubMed] [Google Scholar]

5. Arjomand H, AlSalman J, Azain J, Amin D. Myocardial bridging of left circumflex coronary artery associated with acute myocardial infarction. J Invasive Cardiol. 2000;12:431-4. [PubMed] [Google Scholar]

6. Schar B. [Myocardial bridging - symptoms of coronary disease that sometimes is not]. Praxis (Bern 1994) 2001;90:1923-8. [PubMed] [Google Scholar]

7. Yetman AT, McCrindle BW, MacDonald C, Freedom RM, Gow R. Myocardial bridging in children with hypertrophic cardiomyopathy - a risk factor for sudden death. N Engl J Med. 2004;339:1201-9. [PubMed] [Google Scholar]

8. Gowda RM, Khan IA, Ansari AW, Cohen RA. Acute ST segment elevation myocardial infarction from myocardial bridging of left anterior descending coronary artery. Int J Cardiol. 2003;90:117-8. [PubMed] [Google Scholar]

9. Berry JF, von Mering GO, Schmalfuss C, Hill JA, Kerensky RA. Systolic compression of the left anterior descending coronary artery: A case series, review of the literature, and therapeutic options including stenting. Catheter Cardiovasc Interv. 2002;56:58-63. [PubMed] [Google Scholar]

10. Tio RA, Ebels T. Ventricular septal rupture caused by myocardial bridging. Ann Thorac Surg. 2001;72:136970. [PubMed] [Google Scholar]

11. Feld H, Guadanino V, Hollander G, Greengart A, Lichstein E, Shani J. Exercise-induced ventricular tachycardia in association with a myocardial bridge. Chest 1991;99:1295-6. [PubMed] [Google Scholar]

12. den Dulk K, Brugada P, Braat S, Heddle B, Wellens $\mathrm{HJ}$. Myocardial bridging as a cause of paroxysmal atrioventricular block. J Am Coll Cardiol. 1983;1:9659. [PubMed] [Google Scholar]

13. Galli M, Politi A, Zerboni S. "Functional myocardial bridging" and "hyperkinetic state": A rare association as a cause of acute myocardial infarction. G Ital Cardiol. 1997;27:1286-9. [PubMed] [Google Scholar]

14. Cutler D, Wallace JM. Myocardial bridging in a young 
patient with sudden death. Clin Cardiol. 1997;20:581-3. [PubMed] [Google Scholar]

15. Ferreira AG Jr, Trotter SE, Konig B, Decourt LV, Fox $\mathrm{K}$, Olsen EG. Myocardial bridges: morphological and functional aspects. Br Heart J. 1991;66:364-367. [PubMed] [Google Scholar]

16. Angelni P, Velasco JA, Flamm S. Coronary anomalies: incidence, pathophysiology, and clinical relevance. Circulation. 2002;105:2449-2454. [PubMed] [Google Scholar]

17. Shinjo Sk, Shinjo SMO, Prates NEVB. Bovine Myocardial bridge morphology and association with coronary artherosclerosis. Braz. J Morphol Sci. 2004;24:95-98. [Google Scholar]

18. Saidi HS, Olumbe AO, Kalebi A. The anatomy and pathology of coronary arteries in adult Kenyans. EAMJ. 2002;76:323-7. [Pubmed] [Google Scholar]

19. Bourassa MG, Butnaru A, Lespérance J, Tardif JC. Symptomatic myocardial bridges: overview of ischemic mechanisms and current diagnostic and treatment strategies. J Am Coll Cardiol. 2003; 41:351-9. [Pubmed] [Google Scholar]

20. Erbel R, Rupprecht HJ, Ge J, Gerber T, Gunter G, Meyer J. Coronary artery shape and flow changes induced by myocardial bridging. Assessment by intravascular ultrasound. Echocardiography. 1993; 10:71-77. [Google Scholar]

21. Klues HG, Schwarz ER, vom Dahl J, Reffelmann T, Reul H, Potthast K, Schmitz K, Minartz J, Krebs W, Hanrath P. Disturbed intracoronary hemodynamics in myocardial bridging. Early normalization by intracoronary stent placement. Circulation. 1997;96:2905-13. [PubMed] [Google Scholar]

22. Ge J, Erbel R, Rupprecht HJ, Koch L, Kearney P, Gorge $G$, Haude M, Meyer J. Comparison of intravascular ultrasound and angiography in the assessment of myocardial bridging. Circulation. 1994; 89:1725-1732. [PubMed] [Google Scholar] 